\title{
1 Fragestellungen des Gutachtens
}

Das Gutachten soll Rechtsfragen zur Nutzung von Sozial- und Gesundheitsdaten beantworten, die für das Forschungsprojekt „Smart Analysis - Health Research Access" (SAHRA) von Bedeutung sind. Das Projekt SAHRA wird vom Bundesministerium für Wirtschaft und Energie gefördert. Hauptziel des SAHRA-Projekts ist es, Sozialdaten der AOK Nordost über eine hochsichere web-basierte Datenplattform (SAHRA-Plattform) mit weiteren Datenquellen des Gesundheitswesens zu verbinden. Die Datenplattform soll datenschutzkonforme Möglichkeiten bieten, Abrechnungs-, Behandlungs-, Struktur- sowie Register- und Studiendaten aus verschiedenen Quellen zu kombinieren, zu referenzieren und zu validieren. Die potenziellen Nutzer der SAHRA-Plattform sollen aus der Wissenschaft, der Versorgung und der Industrie stammen.

Durch die grundlegende Änderung des Rechtsrahmens durch die Datenschutz-Grundverordnung der Europäischen Union und die Anpassung mehrerer deutscher Datenschutzregelungen an die neue Rechtslage stellen sich viele grundsätzliche datenschutzrechtliche Fragestellungen in einem neuen Licht. Vor diesem Hintergrund soll in dem Gutachten untersucht werden, wie die folgenden Fragen ${ }^{2}$ nach der zukünftigen Rechtslage, die ab dem 25. Mai 2018 gelten wird, zu beurteilen sind:

2 Der vollständige Fragenkatalog findet sich im Anhang. 
- Unterstützt die Datenschutz-Grundverordnung eher das Konzept eines „absoluten“ oder eines „relativen Personenbezugs“?

- Wird der Begriff der Pseudonymisierung in der Datenschutzgrundverordnung in einer anderen Weise verstanden als in den Vorschriften des Bundesdatenschutzgesetzes und welche Unterschiede folgen daraus, insbesondere für den Prozess der Pseudonymisierung? Welche Bedeutung hat in Bezug auf die bisherige Rechtslage bei der Nutzung pseudonymer Daten das Urteil des Europäischen Gerichtshofs vom 19.10.2016, C - 582/14 (Breyer)?

- Wird der Begriff des Anonymisierens in Erwägungsgrund 26 der Datenschutz-Grundverordnung in einer anderen Weise verstanden als in der bisherigen Definition des Bundesdatenschutzgesetzes und welche Unterschiede folgen daraus bei der Nutzung anonymer Daten für die wissenschaftliche Forschung?

- Kann eine wirksame Anonymisierung von Daten als Umsetzung der Löschpflicht nach Art. 17 Abs. 1 DSGVO angesehen werden? Ist es für eine wirksame Anonymisierung ausreichend, wenn die Löschung im Rahmen einer Einwilligung vereinbart wurde?

- Führt der Begriff der „wissenschaftlichen Forschungszwecke“, wie er in Erwägungsgrund 159 DSGVO verwendet wird, zu einer anderen Interpretation des Begriffs „wissenschaftliche Forschung“, als dies bisher der Fall war? Wie verhält sich der neue Rechtsbegriff zu der Rechtsauffassung von Schneider in der TMF-Veröffentlichung von U.K. Schneider, Sekundärnutzung klinischer Daten - Rechtliche Rahmenbedingungen, Band 12, Seite 97/98?

- Der Bundesrat hat in seinem Beschluss vom 10. März 2017 zum Entwurf der Bundesregierung zu einem Gesetz zur Anpassung des Datenschutzrechts an die Verordnung (EU) 2016/679 und zur Umsetzung der Richtlinie (EU) 2016/680 (Datenschutz-Anpassungs- und -Umsetzungsgesetz EU - DSAnpUG-EU) ${ }^{3}$ die Frage aufgeworfen, inwieweit ein Ausschluss der Auskunftserteilung neben den in $\$ 27$ BDSG-E genannten Voraussetzungen nach objektiven Kriterien auch aus therapeutischen sowie ethischen Erwägungsgründen zum Wohl der betroffenen Person möglich sein sollte. Auf welcher verfassungs- und europarechtlichen Grundlage können solche Ausnahmen von den Auskunftsrechten für wissenschaftliche Forschungsvorhaben vorgenommen werden?

- $\$ 203$ StGB wurde durch das vom Bundestag am 29. Juni 2017 beschlossene Gesetz zur Neuregelung des Schutzes von Geheimnissen bei der Mitwirkung Dritter an der Berufsausübung schweigepflichtiger Personen in der Form geändert, dass die Weitergabe von Informationen an „mitwirkende Personen“ für einen Berufsgeheimnisträger (z.B. Arzt) straflos sein soll, wenn diese an der „beruflichen Tätigkeit“ des Berufsge-

3 BT-Drs. 18/11655, 25, Ziff. 27 lit. d. 
heimnisträgers mitwirken. Kann eine Mitwirkung an einer Forschungstätigkeit eines Arztes auch als Mitwirkungshandlung im Sinn der neuen Rechtsvorschrift verstanden werden? Wie wird die „berufliche Tätigkeit" eines Arztes in der Datenschutz-Grundverordnung im Vergleich zum bisherigen Rechtsrahmen definiert? Gibt es Unterschiede zwischen der ärztlichen Tätigkeit in einem Universitätskrankenhaus, in einem sonstigen Krankenhaus und einer Arztpraxis.

Für alle Fragen soll auch geprüft werden, ob die einschlägigen deutschen Umsetzungsgesetze die Vorgaben aus dem europäischen Primärrecht, dem deutschen Verfassungsrecht und aus der Datenschutz-Grundverordnung einhalten. 